\title{
Über die Schwierigkeit, das Leistungsprinzip im Schulsystem gerechtigkeitstheoretisch zu begründen
}

Replik auf Christian Nerowski (ZfE 2018, 21, 447-464)

\section{Nils Berkemeyer}

Online publiziert: 7. März 2020

(C) Der/die Autor(en) 2020

\section{Die Argumentation Nerowskis}

Nerowskis Beitrag verfolgt die These, dass ,das Problem, für welches das Kriterium ,Leistung' eine Lösung sein kann, die Frage nach der gerechten Verteilung schulischer Zertifikate [ist]“" (Nerowski 2018, S. 443). Ziel des Beitrags sei insgesamt „die Rehabilitierung von ,Leistung“ als relevantem Kriterium im Diskurs um Bildungsgerechtigkeit“" (ebd.). Die Gerechtigkeitsfrage wird somit als Frage der Verteilungsgerechtigkeit interpretiert. Hierfür werden nun zwei Verteilungsgrundsätze angenommen. Zum einen das Bedürfnisprinzip, welches die Verteilung bis hin zu einem Bildungsminimum (bei Nerowski der Hauptschulabschluss) regeln soll, und zweitens das Leistungsprinzip, das die Verteilung aller weiteren Abschlüsse regeln soll, da dessen (Bedürfnisprinzip) ,,mangelnde Aussagekraft oberhalb des Bildungsminimums [verstört]“ (Nerowski 2018, S. 448). Es folgen unterschiedliche Begründungen der These, die institutioneller Art (Leistung zeitigt in der Institution Konsequenzen), anthropologischer Art (Leistung ist eine natürliche Beobachtungskategorie des Menschen in Kooperationssituationen) sowie schultheoretischer Art (zumindest die Oberstufe kann als Zweckverband im Anschluss an Miller interpretiert werden) sind. Nerowski zeigt dann in der Verteidigung seiner These, dass Schülerinnen und Schüler als verantwortlich für ihre Leistungen gedeutet werden können und ihnen darum Leistung auch zurechenbar ist (Voraussetzung für das Verteilungsprinzip Leistung), auch weil die Pädagogik Kinder und Jugendliche als verantwortliche Subjekte adressiert. In einem letzten Schritt grenzt sich Nerowski von der Anerkennungstheorie ab, da ,Inkompatibilitäten“ bestehen (Nerowski 2018, S. 459).

Prof. Dr. N. Berkemeyer $(\bowtie)$

Lehrstuhl für Schulpädagogik und Schulentwicklung, Friedrich-Schiller Universität Jena,

Fürstengraben 11, 07743 Jena, Deutschland

E-Mail: nils.berkemeyer@uni-jena.de 


\section{Zur Problematik der Hauptthese des Beitrags}

Die vorgelegte These ist insgesamt nicht überzeugend, da mit ihr ein Ziel verfolgt wird, welches bereits realisiert ist und ihre Grundidee bereits in Walzers Hauptwerk „Sphären der Gerechtigkeit“ (2006 [1992]), dessen englischsprachiges Original 1983 erschien, formuliert worden ist, ohne dass aber auf Walzer Bezug genommen wird. Zudem finden sich Mängel in der argumentativen Konstruktion.

Die Behauptung, das Leistungsprinzip müsse rehabilitiert werden, muss wenigstens in Bezug auf den Adressaten der Rehabilitierung präzisiert werden. Institutionell ist es verankert und bestimmt weite Teile des Schulalltags und der familialen Diskussion über Schule. Man kann eine Rehabilitation gegenüber spezifischen Vorwürfen vorschlagen, z.B., dass es einen Leistungsmythos gebe (Solga 2013) oder gegenüber anderen verteilungstheoretischen Ansätzen, wie beispielsweise von Rawls (1979), der das Leistungsprinzip nicht für eine gerechte Verteilung von Gütern in der Gesellschaft heranzieht. Nerowski verortet seine Bemühungen aber nicht im gerechtigkeitstheoretischen Diskurs. Hierzu müsste er sich überzeugend von Rawls abgrenzen und deutlich machen, dass Walzer in Deutschland stärker rezipiert werden sollte. Auch müsste deutlich werden, warum, aller empirischen Befunde zum Trotz, das Leistungsprinzip zu verteidigen ist. Nerowski verzichtet auf die Darlegung dieser unterschiedlichen Forschungsstände bzw. auf eine Verortung seiner Position. Hieraus erwächst eine erhebliche Argumentationslücke. Ebenfalls problematisch ist die Ausblendung der Verfahren der Verteilung. Mag das Prinzip auch sinnvoll sein für die Beurteilung der Gerechtigkeit des Schulsystems, so muss es aber auch in Bezug auf die Verfahren der Verteilung diskutiert werden. Dies findet jedoch nicht statt, was die Ausblendung aller Empirie zum Thema ermöglicht (Gomolla und Radtke 2009; Becker und Lauterbach 2016; Autorengruppe Bildungsbericht 2018). Zudem verkürzt Nerowski die Frage nach der Leistungserbringung einseitig auf die Perspektive der Schülerinnen und Schüler. Lernprozesse, und vor allem auch Zertifikate, sind aber durch das Schulsystem (die Lehrkräfte sowie die Angebotsstrukturen) moderiert, das Schulsystem ist somit selbst Leistungserbringer und auf seine Gerechtigkeit hin zu befragen. Dies kommt bei Nerowski nicht in den Blick, weil eine Auseinandersetzung mit Rawls fehlt und somit auch die Idee, Gerechtigkeitstheorien für eine Kritik der gesellschaftlichen Institutionen heranzuziehen. Seine These besagt nur, dass das Prinzip (unabhängig von der Realisierung und den empirischen Befunden) seine Berechtigung hat, um über eine gerechte Verteilung von Gütern zu entscheiden. Damit wird nicht einmal der Diskussionsstand von Walzer (2006) erreicht. Hier hätte man wenigstens Vorschläge zur Optimierung der Anwendung des Leistungsprinzips erwarten dürfen. So wäre dann die Frage in den Blick gekommen, ob Prinzipien der Gerechtigkeit legitimiert werden können, wenngleich die Verfahren und Praktiken der Institution die Prinzipien nicht zur Geltung bringen.

Mit Bezug auf Wigger (2015) verteidigt Nerowski nun nochmals die Fokussierung auf das Leistungsprinzip, da nur so die Verteilungsprobleme im Schulsystem zu bearbeiten seien (Nerowski 2018, S. 433). Dabei zitiert er allerdings jene Passage aus Wiggers Beitrag, die verdeutlichen will, dass solche Teilbetrachtungen einzelner Aspekte der komplexen Problematik, wie bspw. eine losgelöste Diskussion des Leistungsprinzips, genau zu jenen Problemen im Diskurs über Bildungsgerechtig- 
keit führen können, die Nerowski sich hiermit einhandelt. Wigger ist gerade der Auffassung, dass man die Frage der Gerechtigkeit nicht auf das Schulsystem allein verkürzen darf, aber genau dies wird getan; mehr noch, die Frage der Gerechtigkeit wird auf die Frage nach Anschlussoptionen in einem akzeptierten institutionellen Setting verkürzt, in dem Schülerinnen und Schüler für ihren Erfolg verantwortlich sind. Diese Lesart des Schulsystems von Nerowski lässt sich als eine verwaltungstechnische Rechtfertigungstheorie mittlerer Reichweite beschreiben, nicht aber als gerechtigkeitstheoretische Reflexion des Schulsystems klassifizieren. Das sozialkritische Potential von Gerechtigkeitstheorien (Tugendhat 2012), wie es auch von Miller, den Nerowski ja noch als Gewährsmann für seine Argumentation verwendet, wird jedenfalls nicht gesehen oder genutzt. Im Gegenteil, hier wird versucht, aus Aspekten einer kritischen Theorie eine Affirmation institutioneller Verhältnisse abzuleiten.

\section{Der Rückgriff auf das Leistungsprinzip erfolgt auf Basis eines Fehlschlusses}

Es handelt sich bei der Präzisierungsannahme Nerowskis (die Selektionsfunktion zwinge zur Verwendung des Leistungsprinzips) um eine Form des Sein-SollenFehlschlusses (Höffe 2018, S. 31 f.). Aus der Beschreibung des Sachverhalts der Selektion folgt logisch keineswegs, dass man diese nur durch das Leistungsprinzip, ohne Angabe von Verfahren, gerecht lösen kann. Hier werden die institutionellen Setzungen des Schulsystems als invariant betrachtet und darauf aufbauend erfolgt dann die Schlussfolgerung. Moralisch gesehen folgt aber aus der Beschreibung des Schulsystems erst einmal gar nichts. Die Einführung des Verteilungsprinzips „Leistung" muss also auf einem anderen Wege erfolgen.

Selbst wenn man dies als Argument nicht gelten lassen mag, so kann der Begründungsversuch, die gesellschaftlichen Ausgangsbedingungen als Grundlage der moralischen Begründung zu verwenden (Das Schulsystem ist so angelegt, dass es selektieren muss, darum muss das Leistungsprinzip für eine gerechte Selektion sorgen), jedenfalls nicht mit dem Zitat von Taylor - wie es bei Nerowski erfolgt gerechtfertigt werden. Dies wird deutlich, wenn man den Aufsatz Taylors weiterliest und zu folgender Textstelle gelangt:

Solange wir glauben, daß es darum geht, die Prinzipien distributiver Gerechtigkeit zu ermitteln und zu verteidigen, so lange sind nicht nur die Linke und die Rechte auf eine Auseinandersetzung fixiert, für die es keine zufriedenstellende intellektuelle Lösung gibt (Taylor 1992, S. 185; Nerowski zitiert die textgleiche Ausgabe von 1988).

Nerowski tut aber genau das, was Taylor für nicht möglich hält, weshalb Taylor kein geeigneter Kronzeuge für die von ihm vorgelegte Argumentation ist.

Die gütertheoretische Betrachtung ist in der vorgetragenen Form nicht überzeugend, da sie kollaterale Güter (Willke 2014, S. 22) umfasst, die gerade in Bezug auf Leistungen Zurechenbarkeitsprobleme erzeugen. Es ist richtig, dass nicht Bildung als zu verteilendes Gut betrachtet wird (siehe hierzu auch Horster 2015). Aber ob nun Zertifikate, oder auch Unterrichtsberechtigungen, wie an anderer Stelle formu- 
liert, und Einstellungsargumente die richtige Antwort sind, darf ebenfalls bestritten werden. Die Schule wird hier merkwürdig technokratisch und eindimensional gefasst und in ihren tatsächlichen Leistungen und Effekten verkannt. Der vorgetragenen Lesart der Schule ist entgegenzuhalten, dass das, was zunächst - legitimiert durch Gesellschaft - verteilt wird, Schulangebote und Lehrkräfte sind. Diese können einmal auf Geld (Was ist die Gesellschaft bereit für Bildung zu investieren?) zurückgerechnet werden und zudem auf ihre jeweiligen Leistungen, bei Lehrkräften also die Darbietung von Wissen, Beratung, Diagnose, Erziehung u.v.a.m. Die Eingangsbedingungen dieses Distributionsprozesses sind gut überschaubar (Gymnasien sind teuer, Grundschulen sehr günstig), die Prozesse, die im Anschluss an diese „Erstverteilung“ entstehen, sind kaum zu überschauen. Die Ergebnisse sind wiederum gut beobachtbar, auch in Form von Zertifikaten. Diese sind aber eben nicht nur von Leistungen der Schülerinnen und Schüler abhängig, sondern auch vom Schulsystem und den hierin angebotenen Leistungen, seiner äußeren Angebotsstruktur (i. S. der Schulstruktur, siehe hierzu Berkemeyer et al. 2014) und seiner internen Angebotsstruktur (i.S. einer Angebots-Nutzung in Schule und Unterricht, Helmke 2009). Leistung muss entsprechend als Ko-Konstruktion des Schulsystems und seiner Klienten konzipiert und systematisiert werden, nicht nur als Ergebnis einer Schülerhandlung (in Bezug auf Handlungen allgemein wusste dies schon Weber 1976 sehr genau, vgl. Weber 2002).

\section{Mit Miller kann es keine zutreffende Begründung des Leistungsprinzips für das bundesdeutsche Schulsystem geben}

Der Begründungsteil der These orientiert sich ganz wesentlich an Millers Überlegungen in „Grundsätze sozialer Gerechtigkeit“ (Miller 2008). Drei Hauptargumente sprechen gegen die Möglichkeit, das bundesdeutsche Schulsystem mit Miller gerechtigkeitstheoretisch am Leistungsprinzip zu orientieren. Der erste Einwand bezieht sich auf einen von Nerowski nicht aufgelösten Widerspruch, ein zweiter auf einen Kategorienfehler in der Argumentationsstruktur und ein dritter auf eine unvollständige Rezeption des Verdienstprinzips, so wie es von Miller gefasst wird.

1. Die Begründung, warum das Verdienstprinzip durch Leistung als sinnvolles Gerechtigkeitsprinzip zur Anwendung kommen soll, wird durch einen argumentativen Widerspruch eingeleitet (vgl. Nerowski 2018, S. 448 ff.). Zunächst führt Nerowski zwei konkurrierende Argumente für die Verwendung des Verdienstprinzips ins Feld. Zum einen wird institutionell argumentiert (weil es dort schon passiere), zum anderen aber auch vorinstitutionell (es sei ein grundlegender Aspekt in der menschlichen Kooperation, denjenigen Verdienst zukommen zu lassen, die etwas geleistet haben). Miller, auf den er sich bezieht, verweist auf diesen Unterschied:

Entweder behandelt man also Verdienstkriterien als grundlegende Bestandteile einer Gerechtigkeitstheorie und unterstellt damit, dass Verdienst kein bloß institutionelles Konzept ist oder aber man deutet den Begriff in etwas um, das 
sehr viel unbestimmter ist als die übliche Verdienstidee (Miller 2008, S. 189).

Miller entscheidet sich für den vorinstitutionellen Zugang. Nerowski tut hingegen so, als seien beide Varianten für seine Argumentation gleich hilfreich und es bestünde kein Problem (ebenfalls ein Mangel der fehlenden Rawls-Rezeption). Miller weist dem Verdienstbegriff selbst eine kritische Bedeutung zu, da er gerade darauf hinweisen soll, dass eine spezifische Leistung womöglich nicht erbracht wurde. Angesichts der empirischen Befundlage für das Schulsystem könnte man Millers Leistungsbegriff nun nutzen, um das deutsche Schulsystem zu kritisieren, da hier sozialer Status und nicht Leistung Verteilung zumindest moderiert. Nerowski aber will die Praxis der Institution legitimieren und argumentiert nicht im millerschen Sinn vorinstitutionell. Würde man dies tun, könnte man ja fragen, worin die eigentliche Leistung besteht, die als Verteilungskriterium heranzuziehen ist. Man würde dann wohl sehr schnell auf eine intra-individuelle Bezugsnorm kommen, weil letztlich nur in der Beobachtung der individuellen Entwicklung eine persönliche und damit zurechenbare Leistung vermutet werden würde. Anders gefragt: Ist es im moralischen Sinne eine Leistung, die geforderten Standards dauerhaft voll zu erfüllen, ohne sich wesentlich weiterzuentwickeln, weil man besonders intelligent ist? Diese Ebene der Auseinandersetzung wird allerdings nicht erreicht und es kommt $\mathrm{zu}$ der benannten Verwicklung in den nicht erkannten Widerspruch.

2. ,Diejenigen Schülerinnen und Schüler, die in der Schule eine hohe Leistung erbringen, leisten einen hohen Beitrag zum gesamtgesellschaftlichen Wohlergehen. Dieser besteht darin, dass sie sich durch Lernprozesse in die Lage versetzt haben, ihre Kompetenzen in den Dienst der Gesellschaft zu stellen. Sie erwerben damit einen berechtigten und gerechten Anspruch auf eine Vergütung, die von der Schule in Form von weiterführendem Unterricht und gewichtigerer Einstellungsargumente gewährt wird“" (Nerowski 2018, S. 451).

Sehen wir einmal von den problematischen Wertungen des Zitats ab (wir würden doch davon ausgehen, dass alle Menschen einen solchen Beitrag, der wohl kaum nur monetär zu fassen ist, wie, um nur zwei Beispiele zu nennen, die Bedeutung von Erziehrinnen und Erziehern oder Altenpflegerinnen und Altenpflegern für das gesellschaftliche Wohlergehen schnell zeigt), bleibt ein Kategorienfehler, der die Argumentation nicht unerheblich belastet. Nerowski vermischt hier den erwartbaren gesamtgesellschaftlichen Nutzen (ein utilitaristisches Argument) mit dem auf Leistung basierenden Verdienst in Form von Zertifikaten. Richtig bleibt, dass man mit dem Gesamtnutzen argumentieren kann, aber dann muss die Gesamtargumentation auf eine utilitaristische Basis gestellt werden. Aber es ist unzulässig, das Schulsystem innerhalb derselben Argumentation einmal verteilungstheoretisch und ein anders mal utilitaristisch zu rechtfertigen.

3. Die in schultheoretischer Absicht vorgenommene Nutzung der Idee des Zweckverbands bei Miller ist ein interessantes Gedankenexperiment, was allerdings scheitern muss, und nicht wie Nerowski meint, dass Leistungsprinzip zu begründen vermag. Miller hat den Zweckverband als Vergesellschaftungsform konzipiert, in der freie Bürgerinnen und Bürger zum wechselseitigen Vorteil miteinander koope- 
rieren, wobei der Verdienst gemessen an Leistung zur gerechtigkeitstheoretischen Referenzgröße wird (vgl. Nerowski 2018, S. 450 f.). Den Gesellschaftsbereich, der so bei Miller beschrieben ist, würde man wohl als Markt bezeichnen, so wie dies auch in Honneths (2011) Hauptwerk „Das Recht der Freiheit“ zu finden ist. Allerdings geht Miller in der Tat weiter und fasst im Grunde jedwede Organisation, die qua Definition ein Ziel verfolgt, als Zweckverband mit (vertraglich abgesicherter) Mitgliedschaftsoption auf. Und hier beginnt womöglich die Schwierigkeit, die auch Miller nicht hinreichend berücksichtigt, welche nämlich darin besteht, dass neben Zweckverbänden, die quasi eigenständigen Zielen folgen (Geldmaximierung, Titel im Sport, etc.), auch Vergesellschaftungsformen existieren, die Zielsetzungen verfolgen, die quasi außerhalb der Organisation liegen, bzw. die durch die Organisation stellvertretend erfüllt werden. Krankenhäuser sind dergestalt und auch Schulen, denn diese können ihre Ziele nur innerhalb der staatlich vorgegebenen Rahmungen formulieren und verfolgen, und diese sind klarer und enger, als dies mitunter in den Diskussionen über Schulprofilierung erkennbar ist. In Zweckverbänden, in denen die Beziehungen, wie Miller (2008, S. 198) dies ausdrücklich formuliert, instrumenteller Natur sind, wären Schülerinnen und Schüler Vertragspartner, die ihre Leistung ,anbieten“ und im Tausch dafür ein Zertifikat erhalten. Wenn dies zutreffend als empirische Beschreibung der gymnasialen Oberstufe ist, haben wir hier nicht die Rechtfertigung des Leistungsprinzips, sondern die Beschreibung einer Sozialpathologie des Schulsystems. Will Nerowski hier ernsthaft für eine instrumentelle Beziehungskultur in der Oberstufe plädieren, in der sich Schülerinnen und Schüler sowie Lehrkräfte vor allem in Bezug auf ihre Vorteile verhalten und dies dann auch ,,belohnt“ wird? Und auch wenn wir einmal die Idee des Tausches weiterverfolgen würden, wäre schnell klar, dass dieser Tausch kaum gerechtigkeitstheoretischen Ansprüchen genügt, da die vergebenen Zertifikate je nach Bundesland unterschiedlich als Einstellungsargumente gewichtet werden. Es gibt also ein Tauschversprechen, was letztlich gar nicht erfüllt werden kann. Auch dieses Problem müsste einer Lösung zugeführt und diskutiert werden.

Nicht zuletzt sei darauf hingewiesen, dass die Schule multiple Ziele verfolgt man schaue nur einmal in die Schulgesetze -, sodass verschiedenste Leistungen erbracht werden können und mit Verdienst ausgestattet werden müssten. So ist ja die Fähigkeit zur demokratischen Teilhabe an der Gesellschaft das wesentlichste Ziel der Schule und Staatsziel der BRD (GG, Art. 20). Allerdings gibt es hierfür kein Zertifikat und wäre in Nerowskis Lesart darum wohl auch unwichtig oder aber zumindest nicht nützlich. Zudem ist diese Zielerreichung konstitutiv für die Demokratie und kein Ziel, was im Kontext eines Zweckverbands frei wählbar wäre. Problematisch bei Nerowski ist die unspezifische Verwendung des Leistungsbegriffs. Wird dieser nur als Äquivalenz von Benotungen im Kontext von Lernzielkontrollen gesehen, greift dies deutlich zu kurz, eben auch im Sinne der Ziele, die die Schule in der Oberstufe verfolgt. Es soll nicht bestritten werden, dass in der Oberstufe persönlicher Nutzen maximiert werden kann (i.S. von erwartbaren Bildungserträgen, siehe hierzu auch Autorengruppe Bildungsbericht 2018), aber dies umfasst nur einen Teil der schulischen Oberstufe. Das Einüben von Haltungen der Solidarität, die wissenschaftspropädeutische Erkundung von wissenschaftlichen Lebensformen, 
die Pflege von Freundschaften und vor allem die Entwicklung staatsbürgerlicher Tugenden (Sandel 2015) folgen eher einem Kooperationsmodell (siehe hierzu die Beiträge zu Deweys Demokratietheorie in Joas 2000; Nida-Rümelin 2015, 2016). Der Versuch, die Behandlung von Menschen als Mittel zur Zweckerreichung zu legitimieren, muss zurückgewiesen werden und kann für den Bereich der Schule unmöglich unter dem Dach der Gerechtigkeit verhandelt werden (Joas 2000; NidaRümelin 2015, 2016; Knöpfler 2017).

Letztlich liefert aber vermutlich Miller selbst das stärkste Argument, Schule unter aktuellen Bedingungen nicht als Zweckverband zu begreifen. Denn Miller stellt hohe Anforderungen an das Leistungsprinzip, die Nerowski nicht nennt. Miller (2008) führt nämlich ein hartes Kriterium ein, ohne dessen Erfüllung das Leistungsprinzip nicht zur Anwendung kommen sollte - oder es jedenfalls nicht beanspruchen kann, zu einer gerechten Verteilung zu führen: das Äquivalenzpostulat (Miller 2008, S. 189). Gerecht ist eine Verteilung nach dem Leistungsprinzip nur dann, wenn auch für die gleiche Leistung der gleiche Verdienst zugesprochen wird. Dass aber nun in Deutschland dieses Äquivalenzpostulat im Schulsystem als erreicht betrachtet werden kann, kann nun wirklich niemand sinnvollerweise argumentieren, trotz erster Bemühungen um ein bundesweites Zentralabitur. Nerowski erwähnt dieses für seine Argumentation nicht ganz unerhebliche Problem nicht.

\section{Eindimensionale Verantwortungszuschreibungen sind unzureichend}

Nerowski widmet sich in der Verteidigung seiner These vor allem der Frage, ob (1) Schülerinnen und Schüler für ihre Leistungen verantwortlich gemacht werden können, denn nur dann, so seine These, könne man (2) legitimerweise auch die Leistung als Verdienstkriterium heranziehen (vgl. Nerowski 2018, S. 452 ff.).

1. Rawls hatte unter anderem aufgrund der problematischen Zurechenbarkeit von Verdienst davon abgesehen, diesen zu einem allgemeinen Prinzip zu erheben, ohne ihn aber als ein mögliches moralisches Kriterium innerhalb einer gerechten Ordnung zu verwerfen. Nerowski sieht diese Differenz der Ebenen und die Reichweitenunterschiede zwischen allgemeinen Prinzipien und moralischen Kriterien innerhalb der durch die Prinzipien gestalteten Ordnung nicht. Unter Bezug auf einen Beitrag von Strawson, der das metaethische Problem von Determinismus und Indeterminismus verhandelt, kommt er zu dem Schluss, dass es immer nur eine Teilverantwortung gibt, weil man nie voll verantwortlich sein kann. Nerowski trennt hier allerdings nicht zwischen Meta-Ethik und normativer Ethik, was weitreichende Folgen für sein Argument hat. Bereits der bekanntere Namensvetter von Gal Strawson, nämlich Peter Frederick Strawson, hat darauf hingewiesen, dass metaethische Überlegungen zum Determinismusproblem (siehe auch Frankena 2017) keinerlei Auskunft darüber geben können, ob wir uns als wechselseitig frei (und damit verantwortlich) betrachten wollen oder sollen (Willaschek 2003, S. 200) (so übrigens auch die Ausführungen bei Gal Strawson, vgl. auch Höffe 2015, S. 349 ff.). Frankena (2017) ist hinsichtlich der Frage der Zurechenbarkeit von Verantwortung im Übrigen höchst instruktiv, weil deutlich wird, dass es letzt- 
lich um die Frage geht, ob Schülerinnen und Schüler in Bezug auf die Erbringung ihrer Leistung als frei gedeutet werden sollten. Wer das System entlasten will, wird Schülerinnen und Schüler für frei erklären (dies haben ja auch ca. $80 \%$ der Lehrkräfte in der ersten PISA-Untersuchung getan, wenn sie sagen, dass die Schülerinnen und Schüler für ihre Leistung verantwortlich sind), damit zugleich aber eingestehen müssen, dass diese Freiheit in der Schule nur funktional in Bezug auf Verantwortlichkeit gewendet wird. Positive Freiheit meint allerdings, dass Schülerinnen und Schüler auch eine andere Entscheidung hätten treffen können, sie also eine Befähigung dazu hätten haben müssen, sich anders zu entscheiden (Frankena 2017, S. 68). Wenn wir dies gelten lassen, dann macht die Rede von einer moralischen Verantwortung für die eigene Leistung in der Schule eigentlich keinen rechten Sinn. Das philosophische Argument liefert neben Rawls auch Nagel (2015), der die Frage der Verantwortlichkeit schlicht für nicht lösbar hält. Wir sollten von der Zurechnungsspekulation Abstand nehmen. Sie ist für das Geschäft der Schule und die Frage nach der Gerechtigkeit nicht notwendig. Mehr als bestimmte Akteure zu entlasten und andere zu belasten, kann so nicht gewonnen werden.

2. Irritierend sind dann die Argumente, die in Kap. 5.3 vorgetragen werden. Nerowski bringt hier vor, dass das Argument der Nichtzurechenbarkeit von Leistung in den Verantwortungsbereich der Schülerinnen und Schüler (so auch Heinrich 2010 und Stojanov 2011), bereits darum unbegründet sei, dass die Pädagogik doch immer schon dem Subjekt Verantwortung zuschreibt. Nun besteht aber zwischen einer Zuschreibung, die quasi im Erprobungs- und Trainingsmodus der Pädagogik geschieht und einer Zurechnung von Verantwortung zur Herstellung einer gerechten gesellschaftlichen Ordnung ein Unterschied. Hier werden schlicht Bezugstheorien miteinander verwechselt, sodass es zu einem weiteren Kategorienfehler kommt. Die Pädagogik kann zwar als auf der Ethik begründet verstanden werden, aber nicht immer ist das ethische Prinzip der Gerechtigkeit ihr erstes Anliegen. Man könnte sich sogar Situationen vorstellen, in denen sie bewusst ungerecht verfährt, um z. B. Hilfe zu leisten. Nicht jede Hilfeleistung wird zu gerechten Zuständen im Klassenzimmer führen, dennoch bleibt sie als moralisches Prinzip geboten. Aus Zurechnungspraktiken der Pädagogik lässt sich jedenfalls nichts normativ Relevantes über die moralische Verantwortlichkeit von Kindern und Jugendlichen in Bezug auf die von ihnen erbrachte Leistung aussagen.

\section{Gescheiterter Widerspruch: Die Anerkennungstheorie nutzt Leistung explizit}

Die Auseinandersetzung mit der Honneth'schen Anerkennungstheorie ergibt sich keineswegs zwingend aus der Argumentation und wirkt daher am Ende des Beitrags etwas künstlich.

Davon abgesehen ist Nerowskis Versuch, einen Widerspruch zwischen verteilungstheoretischem Gerechtigkeitsprinzip „Leistung“ und der Anerkennungstheorie zu erzeugen, wenig elaboriert. Er formuliert: 
Zwischen Anerkennungstheorie und Leistungsprinzip existieren jedoch Inkompatibilitäten. [...]. Die Ambivalenz von „Leistung“ und „Anerkennung“ liegt nun darin, dass in der Sphäre „Leistung“ “...] nicht nur Anerkennungsgewinner, sondern notwendigerweise auch Anerkennungsverlierer hervorgebracht werden. Schlechte Bewertungen sind die Folge von guten Bewertungen und lassen ein dauerhaftes Potential für Demütigung und Missachtung entstehen. „Leistung“ scheint damit in einem gewissen Widerspruch zu „Anerkennung“ zu stehen (Nerowski 2018, S. 459).

Diese Ausführungen zeigen weniger die Widersprüche als vielmehr die Missverständnisse oder das Nicht-Verständnis der Anerkennungstheorie, wie sie in das „Recht der Freiheit“ entfaltet wird (Honneth 2011). Dass Nerowski auch diesen Beitrag zur Gerechtigkeitstheorie nicht rezipiert und für die Auseinandersetzung heranzieht, obwohl Honneth genau hier die Möglichkeit sozialer Freiheit durch Anerkennung in der Sphäre des Marktes (der einen dem Zweckverband ähnliche Interpretationsraum eröffnet) erörtert, zeigt die Rezeptionslücken. Auch die großen Gemeinsamkeiten zwischen Honneth und Miller, wie sie Honneth im Vorwort zur deutschen Ausgabe von „Grundsätze sozialer Gerechtigkeit“ betont, bleiben von Nerowski unbeachtet, hätten aber wohl eine Erörterung verdient.

\section{Gerechtigkeit als kritische Analysekategorie und Verschiebung der Rechtfertigungsordnung}

Nerowski hat mit seiner Argumentation letztlich versucht, die Idee „,vom Tellerwäscher zum Millionär" für das deutsche Schulsystem zu erzählen und zu begründen. Bereits in der Wahl der Perspektive liegt aber der Ansatzpunkt, andere Fragen zu stellen. Nerowski sieht die Schülerinnen und Schüler in der Verantwortung und darin auch die Lösung für das Problem von Bildungsgerechtigkeit. Vielleicht suggeriert ja bereits der Begriff der Bildungsgerechtigkeit, dass wir bei den Lernsubjekten mit unseren Überlegungen beginnen sollten. Ich halte dies für nicht zwingend und würde auch den Begriff der Bildungsgerechtigkeit vorerst nicht verwenden. Sinnvoller erscheint es zu fragen, was die Schule als Institution zur sozialen Gerechtigkeit (Miller 2008) beitragen kann oder wo sie Ungerechtigkeiten verschärft. Die Schule kann dabei mit Rawls in Bezug auf die gerechte Verfasstheit der „Institution Schule“ theoretisch wie empirisch (Berkemeyer et al. 2017) befragt werden. Darüber hinaus lassen sich die Praktiken innerhalb der Institution Schule anerkennungstheoretisch analysieren, wobei es aber nicht bei der Analyse von Interaktionsbeziehungen belassen werden sollte. Der Sozialtheorie Honneths, genau wie dem Ansatz von Miller auch, geht es um die kritische Analyse von gesellschaftlichen Verhältnissen, auch mit dem Ziel, Sozialpathologien zu identifizieren. Auf das Schulsystem bezogen haben wir dahingehend einen programmatischen Vorschlag unter dem Titel „Kritische Schulsystementwicklungsforschung“" unterbreitet (Berkemeyer et al. im Druck). Es sollten also die Systemleistungen in Bezug auf ihren Beitrag zur sozialen Gerechtigkeit kritisch befragt werden, theoretisch wie empirisch. Dieses kritische Potential steckt in den Gerechtigkeitstheorien und erlaubt es uns normative Regulative in die 
Frage der Schulsystementwicklung einzubringen. Zur Legitimation der bestehenden Verhältnisse taugen sie, wie Miller weiß, wenig. In einer solchen Perspektive wäre dann auch anerkannt, dass Schülerinnen und Schüler einen Anspruch gegenüber der Schule haben. So wird die Aufgabe der Rechtfertigung (Forst 2015) nicht mehr nur den Schülern übertragen, sondern zu einer im besten Sinne gemeinschaftlichen und demokratischen Aufgabe.

Funding Open Access funding provided by Projekt DEAL.

Open Access Dieser Artikel wird unter der Creative Commons Namensnennung 4.0 International Lizenz veröffentlicht, welche die Nutzung, Vervielfältigung, Bearbeitung, Verbreitung und Wiedergabe in jeglichem Medium und Format erlaubt, sofern Sie den/die ursprünglichen Autor(en) und die Quelle ordnungsgemäß nennen, einen Link zur Creative Commons Lizenz beifügen und angeben, ob Änderungen vorgenommen wurden.

Die in diesem Artikel enthaltenen Bilder und sonstiges Drittmaterial unterliegen ebenfalls der genannten Creative Commons Lizenz, sofern sich aus der Abbildungslegende nichts anderes ergibt. Sofern das betreffende Material nicht unter der genannten Creative Commons Lizenz steht und die betreffende Handlung nicht nach gesetzlichen Vorschriften erlaubt ist, ist für die oben aufgeführten Weiterverwendungen des Materials die Einwilligung des jeweiligen Rechteinhabers einzuholen.

Weitere Details zur Lizenz entnehmen Sie bitte der Lizenzinformation auf http://creativecommons.org/ licenses/by/4.0/deed.de.

\section{Literatur}

Autorengruppe Bildungsbericht (2018). Bildung in Deutschland 2018. Ein indikatorengestützter Bericht mit einer Analyse zu Bildung und Migration. Bielefeld: wbv.

Becker, R., \& Lauterbach, W. (2016). Bildung als Privileg - Ursachen, Mechanismen, Prozesse und Wirkungen. In R. Becker \& W. Lauterbach (Hrsg.), Bildung als Privileg. Erklärungen und Befunde zu den Ursachen der Bildungsungleichheit (Bd. 5, S. 3-53). Wiesbaden: Springer VS.

Berkemeyer, N., Bos, W., Hermstein, B., Abendroth, S., \& Semper, I. (2017). Chancenspiegel - eine Zwischenbilanz. Zur Chancengerechtigkeit und Leistungsfähigkeit der deutschen Schulsysteme seit 2002. Gütersloh: Bertelsmann.

Berkemeyer, N., Bos, W., Manitius, V., Hermstein, B., Bonitz, M., \& Semper, I. (2014). Chancenspiegel 2014. Regionale Disparitäten in der Chancengerechtigkeit und Leistungsfähigkeit der deutschen Schulsysteme. Gütersloh: Bertelsmann.

Berkemeyer, N., Hermstein, B., Meißner, S., \& Semper, I. (2019). Kritische Schulsystementwicklungsforschung. Ein normativ-analytischer Forschungsansatz der schulischen Ungleichheitsforschung. JERO, 11(1), 47-73.

Forst, R. (2015). Normativität und Macht. Zur Analyse sozialer Rechtfertigungsordnungen (1. Aufl.). Berlin: Suhrkamp.

Frankena, W. K. (2017). Ethik. Eine analytische Einführung (6. Aufl.). Wiesbaden: Springer VS.

Gomolla, M., \& Radtke, F.-O. (2009). Institutionelle Diskriminierung. Die Herstellung ethnischer Differenz in der Schule (3. Aufl.). Wiesbaden: VS.

Heinrich, M. (2010). Bildungsgerechtigkeit - Zum Problem der Anerkennung fragiler Bildungsprozesse innerhalb neuer Steuerung und demokratischer Governance. In S. Aufenanger, F. Hamburger, L. Ludwig \& R. Tippelt (Hrsg.), Bildung in der Demokratie. Beiträge zum 22. Kongress der Deutschen Gesellschaft für Erziehungswissenschaft (S. 125-144). Opladen: Budrich.

Helmke, A. (2009). Unterrichtsqualität und Lehrerprofessionalität. Diagnose, Evaluation und Verbesserung des Unterrichts; Franz Emanuel Weinert gewidmet (1. Aufl.). Stuttgart: Seelze-Velber.

Höffe, O. (2015). Kritik der Freiheit. München: Beck.

Höffe, O. (2018). Ethik (2. Aufl.). München: C.H.Beck.

Honneth, A. (2011). Das Recht der Freiheit. Grundriß einer demokratischen Sittlichkeit (1. Aufl.). Berlin: Suhrkamp. 
Horster, D. (2015). Bildungsgerechtigkeit aus sozialphilosophischer Sicht. In V. Manitius, B. Hermstein, N. Berkemeyer \& W. Bos (Hrsg.), Zur Gerechtigkeit von Schule. Theorien, Konzepte, Analysen (S. 42-50). Münster: Waxmann.

Joas, H. (Hrsg.). (2000). Philosophie der Demokratie. Beiträge zum Werk von John Dewey (1. Aufl.). Frankfurt a. M.: Suhrkamp.

Knöpfler, N. (2017). Menschenwürde bei Kant. Darmstadt: WBG.

Miller, D. (2008). Grundsätze sozialer Gerechtigkeit. Frankfurt a. M.: Campus.

Nagel, T. (2015). Der Blick von nirgendwo (2. Aufl.). Frankfurt a. M.: Suhrkamp.

Nerowski, C. (2018). Leistung als Kriterium von Bildungsgerechtigkeit. Zeitschrift für Erziehungswissenschaft, 21(3), 441-464.

Nida-Rümelin, J. (2015). Die Optimierungsfalle. Philosophie einer humanen Ökonomie. München: btb. Genehmigte Taschenbuchausgabe

Nida-Rümelin, J. (2016). Demokratie als Kooperation (2. Aufl.). Frankfurt a. M.: Suhrkamp.

Rawls, J. (1979). Eine Theorie der Gerechtigkeit (1. Aufl.). Frankfurt a. M.: Suhrkamp.

Sandel, M. J. (2015). Gerechtigkeit. Wie wir das Richtige tun (3. Aufl.). Berlin: Ullstein Taschenbuch.

Solga, H. (2013). Meritokratie - die moderne Legitimation ungleicher Bildungschancen. In P. A. Berger \& H. Kahlert (Hrsg.), Wie das Bildungswesen Chancen blockiert (3. Aufl., S. 19-37). Weinheim: Juventa-Verl.

Stojanov, K. (2011). Bildungsgerechtigkeit. Rekonstruktionen eines umkämpften Begriffs (1. Aufl.). Wiesbaden: VS.

Taylor, C. (1992). Negative Freiheit? Zur Kritik des neuzeitlichen Individualismus (1. Aufl.). Frankfurt a. M.: Suhrkamp.

Tugendhat, E. (2012). Vorlesungen über Ethik (8. Aufl.). Frankfurt a. M.: Suhrkamp.

Walzer, M. (2006). Sphären der Gerechtigkeit. Ein Plädoyer für Pluralität und Gleichheit. Frankfurt: Campus.

Weber, M. (2002). Wirtschaft und Gesellschaft. Grundriss der verstehenden Soziologie. Bd. 5. Tübingen: Mohr-Siebeck.

Wigger, L. (2015). Bildung und Gerechtigkeit - Eine Kritik des Diskurses um Bildungsgerechtigkeit aus bildungstheoretischer Sicht. In V. Manitius, B. Hermstein, N. Berkemeyer \& W. Bos (Hrsg.), Zur Gerechtigkeit von Schule. Theorien, Konzepte, Analysen (S. 72-92). Münster: Waxmann.

Willaschek, M. (2003). Freiheit als Bedingung für Verantwortung. Ein kurzes Argument für den Kompatibilismus. In S. Mischer (Hrsg.), Metaphysische und ethische Annäherungen an die menschliche Freiheit (S. 199-206). Münster: LIT.

Willke, H. (2014). Regieren. Politische Steuerung komplexer Gesellschaften. Berlin: Springer. 